\title{
Accessibility, Use and Effectiveness of Neurotechnology Devices for Improved Productivity in Workplace.
}

\author{
Aliyu Aminu Ahmed*, Rukayya Aminu Muhammed ${ }^{* *}$ \\ * Evaluation Department, Monitoring and Evaluation Institute, Abuja www.mandeinstitute.com \\ mandetrainer@gmail.com +2348056155435
}

DOI: 10.29322/IJSRP.11.10.2021.p11803

http://dx.doi.org/10.29322/IJSRP.11.10.2021.p11803

\begin{abstract}
Technological advancement from the beginning of this new millennium has been unmatched since the history of humanity. Humanity has leaped into to new frontiers in information and communication technologies. One of the new frontiers in science is Neurotechnology. Neurotechnology is a multibillion-dollar industry that is exponentially growing and giving science the opportunity to effectively explore the human brain. The proliferation and interest in Neurotechnology devices make it no longer feasible to confine their use to laboratories, they are now sold directly to the customers (DTC). Neurotechnologies were primarily used in medical field and now gradually introduced to other areas. While these technologies have demonstrated significant benefits to the medical field as relates to enhancement of physical and cognitive capabilities, their application in human resource management might raise several ethical, legal, social, and human rights implications. This study used a qualitative approach of systematic literature review as a methodology to explore the accessibility and use of the neurotechnology devices towards improving human productivity in the workplace. The study gave insights on the long-term implications of the neurotechnological devices as relate to the issue of safety, protection, and privacy. There is a possibility that manufacturers of the devices are motivated by profits and not safety of the consumers. It is therefore necessary for consumers and policy makers to understand the neurotechnology landscape, use and ethical challenges at this early stage of development and introduction to the public. It is necessary to design adequate and timely regulatory standards for Neurotechnologies that will enhance policy formulation in the field.
\end{abstract}

Index Terms- Neurotechnology, Neuroscience, Neuroeconomics, Neurotechnology devices. 


\section{Neuroscience and Neurotechnology}

The word neuro means nerve and nervous system ("Brain \& Spine Foundation | What is a neurological problem?", 2021). Neuroscience is defined as the study of how the nervous system develops, its structure, and what it does (Brazier, 2018). The study of Neuroscience has many branches which includes amongst others; Affective neuroscience, Behavioral neuroscience, Clinical neuroscience, Cognitive neuroscience, Computational neuroscience, Cultural neuroscience, Developmental neuroscience, Molecular and cellular neuroscience, Neuro-engineering, Neuroimaging , Neuro-informatics, Neurolinguistics and Neurophysiology(Brazier, 2018). Neuroscience is an emerging field potential of which are exploited within economics.

Neuroeconomics is an interdisciplinary field in economics that is related to neuroscience, and it focuses on the brain's work when humans make decisions [economic decisions] (Ogaki \& Tanaka, 2017).

The emergence of Neuroscience and Neuroeconomics has contributed to exploration into new frontiers for both organizations that produce the technologies that are based on it, as well organizations that want to take the opportunities of these products to maximize and optimize their services and consequently profitability.

Neurotechnology is an aspect of Neuroscience that can be defined as the assembly of methods and instruments to enable direct connection of technical components [computer-based devices] with the nervous system (Müller \& Rotter, 2017). The technologies produced by Neuroscience are called, "Neurotechnologies" or "Neurotechnology devices". Neurotechnologies are mostly found in the medical profession. Consequently, these devices are mainly considered as medical devices that interfere with the central and peripheral nervous system (Anderson et al., 2021). The neurotechnology devices work by applying electrical or magnetic fields through the scalp to change human brain without the need for invasive procedures (National Academies of Sciences, Engineering, and Medicine, 2017). These devices monitor, coordinate, and change brain activity (Lacková \& Kováčová, 2021).

The changes in the brain activity are intended to be beneficial to the consumers who are in many cases medical patients or research volunteers in laboratories working to contribute breaking new frontiers in neurotechnology.

\section{Neurotechnology Access and Use}

People have access to several technologies around them for centuries, these technologies are used for numerous purposes. The quality of life of human beings have greatly been enhanced by technology and technological devices that boosts our abilities to solve problems faster, fight diseases better, and meet our basic needs in an efficient and cost-effective manner (Stieglitz, 2019). Neurotechnology devices are accessible to organizations and people that want and can afford. Neurotechnologies can be procured and used purely for research purposes or in practical applications to influence the brain or nervous system ("Neurotechnologies: The Next Technology Frontier", 2021). Some of these technologies are accessible to the public and consumers use online platforms to directly purchase the devices.

Neurotechnology products have huge and expanding market that is valued approximately \$9.1 billion and will grow approximately $\$ 15.1$ billion by 2024 (Minielly et al., 2020). There is increasing production and use of innovative technologies in neuroscience field. According to Wexler \& Reiner (2019) neurotechnology devices are being sold directly to consumers (DTC) without necessitating the physician as an intermediary.

It can be argued that Neurotechnology, and other related technological devices are designed and sold with a purpose to enhance services, performance, and general improvement of humanity. The contribution of neurotechnology to improvement of humanity is majorly noticed in the areas of medicine, where certain disabilities and disorders are treated. These devices have already been widely used to improve cognitive and motor abilities. Yuste, 2021). Neurotechnology-based interference with brain activity can be very effective, allowing for successful treatment of brain disorders (Müller \& Rotter, 2017). Neurotechnologies are also significantly used to treat neurodegenerative diseases. For example, individuals that have impairment in their five senses (especially sight, sound, and touch) are now treated successfully using neurotechnology devices. 
The use of neurotechnology devices is prevalent in the medical and wellness industries and the devices are increasingly being piloted in education, workplace management, national security, and sports. Neurotechnologies have potentials to read the mind, cure paralysis, restore senses, and more ("Four Great Landmarks in the History of Neurotechnology | Open Mind", 2021).

\section{Neurotechnology and Workplace}

The use of Neurotechnologies in the work place are to support learning and enhance efficiency of the workforce ("Neurotechnologies: The Next Technology Frontier", 2021). There is evidence that neurotechnology devices can provide greater understanding and access to core mechanisms that underlie human identify, memories, personalities, and mind (Goering \& Yuste, 2021). Consequently, there is great potentials for understanding and manipulating employee performance.

\section{Neurotechnology Improvements}

The production and successful application of neurotechnological devices started in 1980s with mostly experimental and therapeutic use in the field of neural medicine (Lacková \& Kováčová, 2021). Since the early production of the neurotechnology devices there has been improvement compared to the present generation of devices mostly presented as prototypes but could not be classified as safe and functional.

Neurotechnology devices have been improved in designs and functionality. These devices are now gradually becoming more appealing, more accessible to the public and sold directly to consumers (Coates McCall, Lau, Minielly \& Illes, 2019).

The advancement in information and communication technologies has contributed greatly in the way these technologies are produced, marketed, and used by consumers. The increasing interest in the technologies is obvious in the level of crowdfunding supporting their development.

\section{Neurotechnology and Ethics}

There has been increasing interest in studying the brain and neural networks. The studies of the brain and use of the Neurotechnologies have traditionally been associated with research institutions, medicine, and medical related professions. Consequently, devices developed in the field of neurotechnology are known to be mostly limited to research and medical purposes. However, these devices are gradually being introduced to other fields of human endeavors such as education, sports, and workplace management. While there has been considerable progress in neurotechnology research across the world using powerful methods, but all these are at experimentation level, and some are indeed unlikely to be applied to humans soon (Goering \& Yuste, 2021).

Neurotechnology devices accessibility and use in research, medical and related fields across the world have limited ethical and legal structures (National Academies of Sciences, Engineering, and Medicine, 2017), therefore the use of neurotechnology devices in the work place is not an exception. Also, there seem to be no clear universally acceptable standards, guidelines as relates to ethics and legalization of the devices. Also as regards human rights, the issue of neurotechnology "largely remains a terra incognita for human rights law" (Ienca \& Andorno, 2017)

\section{Neurotechnology and Safety Concerns}

Like every other technology, neurotechnology have demonstrated a lot of potentials as well obvious challenges. The challenges of Neurotechnology are mainly around the concerns of safety and security of its use or application as most of these technologies require direct contact with the body and in some cases implantation which could trigger defensive response by the human body beyond basic trauma of insertion. There could also be concerns as relate to the type of materials used in producing these technologies (Stieglitz, 2019), considering that in many instances signals are sent to several neural networks in the body. There is limited understanding of the long-term impact of use of neurotechnology devices in the human body. A lot of innovative neurotechnology devices are only at experimentation and not widely approved by regulatory agencies for public use. 


\section{Methodology}

A research method is a strategy of enquiry which moves from the underlying philosophical assumption to the research design and data collection' (Myers and Avison, 2002;lacono, et al 2009 ). There are several considerations in choosing methods of gathering information, this includes costs, time, degree of rigor, who will utilize the findings and others (Walle, 2015). The approaches to be used in a study depends on the purpose of the study. A literature review can be qualitative, quantitative or mix design of the two. The literature review methodology has various approaches and guidelines. The rationale for selecting qualitative method for review is that the strict systematic literature review process is applied to collect the articles and qualitative approach is used to assess them (Snyder, 2019). The study chose a qualitative approach of systematic literature review as a methodology to analyze significant available evidence and publications in a systematic, robust, and objective manner from relevant electronic databases (Bruce \& Mollison, 2004). By using systematic literature review as a research methodology several findings and perspectives from many high-quality empirical findings can be integrated to address the research questions set by this study with a power that no single research has (Snyder, 2019).

\section{Research Questions}

The study attempts to contribute to answering the following questions:

- What are the potential privacy concerns on sell of neurotechnology devices directly to consumers (DTC)?

- Does the use of neurotechnology in the workplace infringe on the fundamental rights of employees?

\section{Limitation of the Study}

The following are the limitations of the study.

(a) Methodological: As with all other qualitative researchers, this study relied on the researcher as key instrument in the data collection and analysis (Myburgh \& Poggenpoel, 2007). Like many structured literature reviews there was limitation in selection of suitable search terms, selecting appropriate databases and deciding on inclusion and exclusion criteria and having too many articles to review (Snyder, 2019). The study used secondary data from diverse literature, mostly online sources. Therefore, opinions may support and sometimes conflict each other.

(b) Logistical: The research was intended to be of exploratory nature hence the logistical Limitation was basically of time within which to implement a more complete and rigorous study.

\section{Theoretical Framework}

Neuroscience studies the complex nervous system. The most important goal of neuroscience is to establish precise structurefunction relationships in the brain (Oktar, 2006). The overarching goal of theory, modelling and statistics in neuroscience is to create an understanding of how the brain works (Jorgenson et al., 2015). In discussing the theories in the field of neuroscience we can look at the four basic concepts on neuroscience which are consciousness, perception, cognition, and behavior (Oktar, 2006). These four concepts have complex, complementing and sometimes diverging theories as relates to neurotechnology, and the use and functioning of neurotechnology devices. According to Oktar (2006), Consciousness is unique and exclusive to everyone. Within everyone also, consciousness is dynamic. Also, perception depends on representation. Representationists theories hold that representations are the brain's symbolic descriptions or interpretations of the world, from which the brain draws inferences about what is there. In neuroscience cognition relates to the working memory. There are several theories relating to cognition such as cognitive map theory, standard consolidation theory and multiple trace theory (Oktar, 2006).

Drawing on the insights from disability and critical disability theory (Gillies, 2014), it can be argued that Neurotechnologies provide agential assistance as caregivers (Goering et al., 2021). However, in the work place not all the workers are disabled therefore use of Neurotechnologies could not be considered as a necessity as the neuros in the brain are characterized by ultra-high complexity of structural connectivity, all of which change and evolve in response to experience (Wang et al., 2020).

\section{Systematic Literature Review Method}

The methodology used for this study is literature review of peer reviewed articles and other online sources. The literature review methodology explores diverse perspectives from many studies and authors which gives a wider understanding of the subject or issue that is studied (Snyder (2019). Literature review involves several chronological steps that includes selecting the topic, developing the argument, searching the literature, surveying the literature, critiquing the literature, and writing the review (Machi, Lawrence A., and Brenda T. McEvoy, 2016). Literature reviews can be of different types such narrative reviews, integrative reviews, systematic reviews and so on. This study deployed a systematic approach of the literature review asking specific questions, synthesizing, and comparing evidence (Snyder (2019). According to Bruce \& Mollison (2004), the systematic approach includes asking and classifying the research questions, identification of eligible literature, assessment of study quality, data extraction, summarizing and interpreting the results. 
Below is a diagram depicting the process used during the literature review. The diagram was adapted and modified from Bruce \& Mollison (2004) and Mengist et al., (2020). As a preliminary activity the study accessed three databases: (I) Science Direct open access, (ii) Semantic Scholar and (iii) Google Scholar. The search strategy was conducted using the Boolean operator "and" between the two key words "Neurotechnology" and "devices" as well a phrase in quotation marks without the Boolean operator. An additional strategy was further adapted; (A) Science Direct only articles that were open access and Portable Document Format (PDF) were selected. (B) Semantic Scholar a filtering strategy was used to identify only articles that were published in 2021 in the fields of business and economics and has PDF were selected. (C) The Google Scholar all the search results for the phrase "Neurotechnology devices" were considered, out of which those with PDF versions were selected.

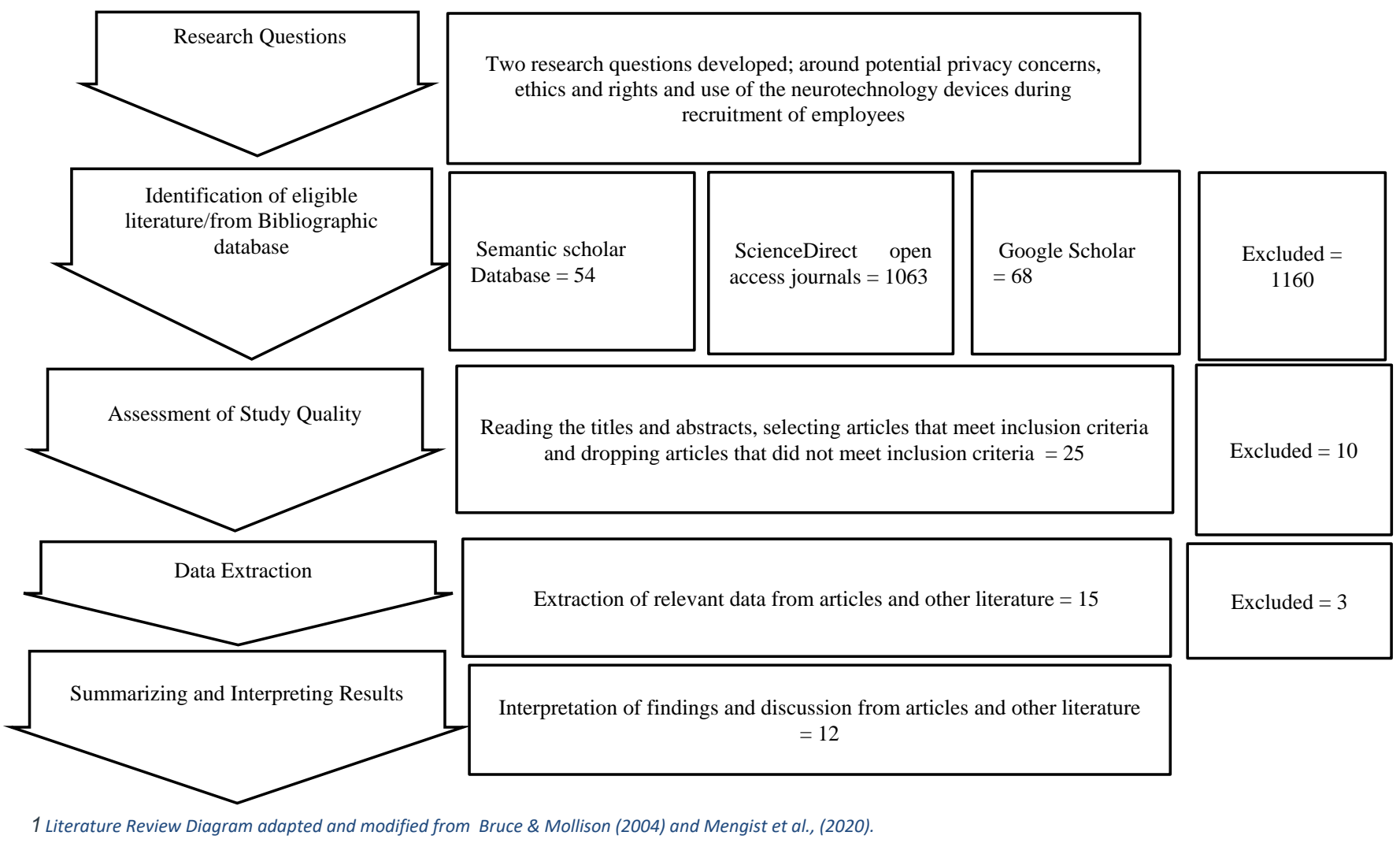

\section{FINDINGS}

Neurotechnology is a complex and emerging field that try to connect the activities and processes in the human brain (nervous system) with machines. The field of neurotechnology is interdisciplinary and controversial, as one of its goals is to directly "wire up" the human brains to machines (Müller \& Rotter, 2017) and modify electrical activity of the nervous system for improved physical and cognitive performance (Khan \& Aziz, 2019). Despite the complexities associated with these neurotechnology devices they are now marketed and sold directly to consumers. The production and use of Neurotechnologies is increasing. There exist significant ethical , legal, and social concerns on widespread use of the Neurotechnologies and this has potentials to affect individual and collective sense of privacy, autonomy and identify (Fuchs, 2006). Despite the interest in neurotechnology the understanding of how it is used in the work place is limited. There is a possibility that companies that manufacture and market these neurotechnology devices are motivated by profit, not goodness (Wexler, 2019).

Neurotechnologies are marketed in the following major product categories; neuromonitoring devices, cognitive training applications, neurostimulation devices, and mental health apps (Wexler \& Reiner, 2019). These devices are sold online and accessible to all consumers. Neuromonitoring devices that are marketed online can be used for monitoring of neurophysiological functions of the central and peripheral nervous system (Intraoperative Neuromonitoring, 2021). Cognitive training applications such as Cognitive Mobile Games (CMG) have been in existence for several decades and are used to training and challenging the brain, they are found to be useful in improving cognitive performance in both young and old adults (Bonnechère et al., 2021). Neurostimulation devices are implantable and programable medical devices that deliver electrical stimulation to specific parts of the brain to treat diseases (Therapeutic Goods Administration , 2021). Neurostimulation therapies can include invasive and noninvasive approaches (Edwards et al., 2017). There are varieties of mental health apps available free ("Mental health apps - NHS", 2021) and for a price ("The Best Mental Help Apps , 2021). While these mental health apps are not designed for diagnoses of mental conditions, but they can help improve mental health ("The 10

This publication is licensed under Creative Commons Attribution CC BY.

http://dx.doi.org/10.29322/IJSRP.11.10.2021.p11803

WwW.ijsrp.org 
Best Mental Health Apps of 2021", 2021) . In the medical field, already ultra-tiny smartphone-controlled, wirelessly rechargeable soft brain implants have been developed for long-term neural circuit manipulation (Kim et al., 2021).

The workplace is a crammed with software and devices that are aimed at managing employees. There are numerous employee management software solutions (Regan, 2021) most of the software are used for mobile employee management, risk assessment, finance management, process management, vendor and staff onboarding and recruitment, employee verification, and/or performance evaluations. Also, biometric scanners and fingerprint identification systems are widely being used in workplace (Jaco, 2021). Most recently the workplace has started using NFC chip implants that have not been certified by any government regulatory agency for use in human body. The NFC chip implants were originally used as alternatives for tags on livestock but now is being experimented on workers in the workplace to enable commercial access control systems ("xNT NFC Chip", 2021). These software and devices are sufficient to manage the work place. Adding neurotechnology devices to the mix will only complicate matters and infringe on the rights of the employees while exposing them to risks of manipulation and exposure to privacy malpractices by their supervisors and the corporate world.

\section{RECOMMENDATIONS}

Neurotechnologies and other technologies have contributed to enhancement of human physical and cognitive capabilities. These technologies are used to read signals from the brain, the technologies can as well stimulate the brain (Coates McCall, Lau, Minielly \& Illes, 2019) and interface with the nervous system of the human body (Stieglitz, 2019). There is need for consumers and policy makers to understand the neurotechnology landscape, use and ethical challenges of these technologies at this early stage of development and introduction to the public. It is also necessary to identify and document all possible implications on accessibility, use and effectiveness of neurotechnology devices in the work place to help enhance product development and protect the labor force. It is necessary to design adequate and timely regulatory standards that will enhance policy formulation in the field of neurotechnology. The sale of neurotechnology devices directly to consumers (DTC) should be regulated and monitored. It is not necessary to use neurotechnology devices during probationary period of workers nor for measuring performance of employees as employees.

\section{CONCLUSION}

Neurotechnology is a multibillion-dollar industry that is exponentially growing and has demonstrated potentials to benefit humanity. The accessibility and use of Neurotechnologies are mostly found in the medical profession and research outfits because they interfere with the highly complex organ in the human body. Despite the potential benefits there are obvious concerns in their use in the work place to improve productivity, these concerns are mainly around privacy, ethics, and human rights. There is a possibility that manufacturers of the devices are motivated by profits that could explain the reason for selling these devices directly to consumers. It is therefore necessary to understand the neurotechnology landscape with the view to designing adequate and timely regulatory standards and will enhance policy formulation in the field.

This publication is licensed under Creative Commons Attribution CC BY.

http://dx.doi.org/10.29322/IJSRP.11.10.2021.p11803

WwW.ijsrp.org 


\section{REFERENCES}

[1] Anderson, L., Antkowiak, P., Asefa, A., Ballard, A., Bansal, T., \& Bello, A. et al. (2021). FDA Regulation of Neurological and Physical Medicine Devices: Access to Safe and Effective Neurotechnologies for All Americans. Retrieved 9 September 2021. http://dx.doi.org/10.1016/j.neuron.2016.10.036

[2] Bonnechère, B., Klass, M., Langley, C., \& Sahakian, B. (2021). Brain training using cognitive apps can improve cognitive performance and processing speed in older adults. Scientific Reports, 11(1). https://doi.org/10.1038/s41598-021-91867-Z

[3] Brain \& Spine Foundation | What is a neurological problem?. Brainandspine.org.uk. (2021). Retrieved 10 September 2021, from https://www.brainandspine.org.uk/information-and-support/what-is-a-neurological-problem

[4] Brazier, Y. (2018). Neuroscience: Overview, history, major branches. Retrieved 1 September 2021, from https://www.medicalnewstoday.com/articles/248680

[5] Bruce, J., \& Mollison, J. (2004). Reviewing the literature: adopting a systematic approach. Journal Of Family Planning and Reproductive Health Care, 30(1), 1316. doi: $10.1783 / 147118904322701901$

[6] Coates McCall, I., Lau, C., Minielly, N., \& Illes, J. (2019). Owning Ethical Innovation: Claims about Commercial Wearable Brain Technologies. Neuron, 102(4) 728-731. doi: 10.1016/j.neuron.2019.03.026

[7] Edwards, C., Kouzani, A., Lee, K., \& Ross, E. (2017). Neurostimulation Devices for the Treatment of Neurologic Disorders. Mayo Clinic Proceedings, 92(9), 14271444. https://doi.org/10.1016/j.mayocp.2017.05.005

[8] Four Great Landmarks in the History of Neurotechnology | OpenMind. OpenMind. (2021). Retrieved 14 September 2021, from https://www.bbvaopenmind.com/en/science/bioscience/four-great-landmarks-in-the-history-of-neurotechnology/

[9] Gillies, J. (2014). Critical Disability Theory. Encyclopedia Of Quality of Life and Well-Being Research, 1348-1350. https://doi.org/10.1007/978-94-007-0753$\underline{5 \_619}$

[10] Goering, S., \& Yuste, R. (2021). On the Necessity of Ethical Guidelines for Novel Neurotechnologies. Retrieved 9 September 2021. Cell. http://dx.doi.org/10.1016/j.cell.2016.10.029

[11] Goering, S., Brown, T., \& Klein, E. (2021). Neurotechnology ethics and relational agency. Philosophy Compass, 16(4). https://doi.org/10.1111/phc3.12734

[12] Google Scholar (2021). Retrieved 9 September 2021, from https://scholar.google.com/

[13] Ienca, M., \& Andorno, R. (2017). Towards new human rights in the age of neuroscience and neurotechnology. Life Sciences, Society And Policy, 13(1). https://doi.org/10.1186/s40504-017-0050-1

[14] Intraoperative Neuromonitoring (2021). Safe and secure with systems from inomed. En.inomed.com Retrieved 14 September 2021 , from https://www.en.inomed.com/products/intraoperative-neuromonitoring-ionm/

[15] Jaco, M. (2021). Rmagazine.com. Retrieved 14 September 2021, from https://rmagazine.com/articles/biometric-scanners-fingerprint-identification-in-theworkplace.html.

[16] Jorgenson, L., Newsome, W., Anderson, D., Bargmann, C., Brown, E., \& Deisseroth, K. et al. (2015). The BRAIN Initiative: developing technology to catalyse neuroscience discovery. Philosophical Transactions of The Royal Society B: Biological Sciences, 370(1668), 20140164. https://doi.org/10.1098/rstb.2014.0164

[17] Kim, C., Ku, M., Qazi, R., Nam, H., Park, J., \& Nam, K. et al. (2021). Soft subdermal implant capable of wireless battery charging and programmable controls for applications in optogenetics. Nature Communications, 12(1). https://doi.org/10.1038/s41467-020-20803-y

[18] Lacková, I., \& Kováčová, M. (2021). Assessment of Neurotechnologies: The Quest For Social And Personal Security. Stumejournals.com. Retrieved 9 September 2021, from https://stumejournals.com/journals/confsec/2019/4/132

[19] Machi, Lawrence A., and Brenda T. McEvoy. The Literature Review : Six Steps to Success. Third ed. 2016.

[20] Mengist, W., Soromessa, T., \& Legese, G. (2020). Method for conducting systematic literature review and meta-analysis for environmental science research. Methodsx, 7, 100777. https://doi.org/10.1016/j.mex.2019.100777

[21] Mental health apps - NHS. nhs.uk. (2021). Retrieved 14 September 2021, from https://www.nhs.uk/apps-library/category/mental-health/

[22] Minielly, N., Hrincu, V., \& Illes, J. (2020). Privacy Challenges to the Democratization of Brain Data. Iscience, 23(6), 101134 https://doi.org/10.1016/j.isci.2020.101134

[23] Müller, O., \& Rotter, S. (2017). Neurotechnology: Current Developments and Ethical Issues. Frontiers In Systems Neuroscience, 11. https://doi.org/10.3389/fnsys.2017.00093

[24] Myburgh, C., \& Poggenpoel, M. (2007). Qualitative methods: a research approach worth considering. Ajol.info. Retrieved 11 September 2021, from https://www.ajol.info/index.php/ajpsy/article/view/30235/5716

[25] Myers, M.D. and Avison, D. (Eds.) (2002) Qualitative Research in Information Systems London: Sage.

[26] National Academies of Sciences, Engineering, and Medicine. (2017). International Perspectives on Integrating Ethical, Legal, and Social Considerations into the Development of Non-Invasive Neuromodulation Devices: Proceedings of a Workshop-in Brief. Washington, DC: The National Academies Press. https://doi.org/10.17226/24643

[27] Neurotechnologies: The Next Technology Frontier. IEEE Brain. (2021). Retrieved 14 September 2021, from https://brain.ieee.org/topics/neurotechnologies-thenext-technology-frontier/.

[28] Ogaki, M., \& Tanaka, S. (2017). What Is Neuroeconomics?. Behavioral Economics, 23-30. https://doi.org/10.1007/978-981-10-6439-5_2

[29] Oktar N., (2006) Theory of Neuroscience Article • January 2006 Journal of Neurological Sciences [Turkish] 23:(3)\# 8;155-158, 2006 http://www.jns.dergisi.org/text.php3?id=107

[30] Regan, R. (2021). Top 12 Employee Management Software Solutions. Connecteam. Retrieved 14 September 2021, from https://connecteam.com/top-10-employeemanagement-tools/.

[31] Science Direct open access (2021). Retrieved 9 September 2021, from https://www.sciencedirect.com/\#open-access

[32] Semantic scholar (2021), Retrieved 9 September 2021, from https://www.semanticscholar.org/about

[33] Snyder, H. (2019). Literature review as a research methodology: An overview and guidelines. Journal Of Business Research, 104, 333-339. doi: 10.1016/j.jbusres.2019.07.039

[34] Stieglitz, T. (2019). Why Neurotechnologies? About the Purposes, Opportunities and Limitations of Neurotechnologies in Clinical Applications. Neuroethics, 14(1), 5-16. doi: 10.1007/s12152-019-09406-7

This publication is licensed under Creative Commons Attribution CC BY.

http://dx.doi.org/10.29322/IJSRP.11.10.2021.p11803

WWW.ijsrp.org 
ISSN 2250-3153

[35] The 10 Best Mental Health Apps of 2021. Healthline. (2021). Retrieved 14 September 2021, from https://www.healthline.com/health/mental-health/mental-healthapps\#what-are-mental-health-apps

[36] The Best Mental Help Apps. (2021). Retrieved 14 September 2021, from https://www.prevention.com/health/mental-health/g33447737/best-apps-for-mental-health/

[37] Therapeutic Goods Administration (TGA). (2021). Medtronic neurostimulation devices used for deep brain, spinal cord and peripheral nerve stimulation therapies - multiple models. Retrieved 14 September 2021, from https://www.tga.gov.au/alert/medtronic-neurostimulation-devices-used-deep-brain-spinal-cord-andperipheral-nerve-stimulation-therapies-multiple-models

[38] Walle, A. (2015). Qualitative Research in Business. Cambridge Scholars Pub.

[39] Wang, X., Hu, H., Huang, C., Kennedy, H., Li, C., \& Logothetis, N. et al. (2020). Computational neuroscience: a frontier of the 21st century. National Science Review, 7(9), 1418-1422. https://doi.org/10.1093/nsr/nwaa129

[40] Wexler, A. (2019). Direct-to-Consumer Neurotechnology: A Grounded Appraisal. AJOB Neuroscience, 10(4), 172-174. https://doi.org/10.1080/21507740.2019.1665138

[41] Wexler, A., \& Reiner, P. (2019). Oversight of direct-to-consumer neurotechnologies. Science, 363(6424), 234-235. https://doi.org/10.1126/science.aav0223

[42] xNT NFC Chip | RFID \& NFC Chip Implants and Biohacking products. Dangerous Things. (2021). Retrieved 14 September 2021, from https://dangerousthings.com/product/xnt/. 